\title{
Nucleating Effect of Carbon Nanoparticles and Their Influence on the Thermal and Chemical Stability of Polypropylene
}

\author{
F. Avalos-Belmontes, ${ }^{1}$ L. F. Ramos-deValle, ${ }^{2}$ E. Ramírez-Vargas, ${ }^{2}$ S. Sánchez-Valdes, ${ }^{2}$ \\ J. Méndez-Nonel, ${ }^{2}$ and R. Zitzumbo-Guzmán ${ }^{3}$ \\ ${ }^{1}$ Departamento de Polímeros, Facultad de Ciencias Químicas (FCQ), Universidad Autónoma de Coahuila (UAdeC), \\ Avenida V. Carranza S.N., 25000 Saltillo, COAH, Mexico \\ ${ }^{2}$ Departamento de Procesamiento de Polímeros, Centro de Investigación en Química Aplicada (CIQA), \\ Bulevar, Enrique Reyna 140, 25253 Saltillo, COAH, Mexico \\ ${ }^{3}$ Departamento de Materiales, CIATEC, A.C. Omega 201, Fracc. Delta, 37545 León, GTO, Mexico
}

Correspondence should be addressed to F. Avalos-Belmontes, favalos@uadec.edu.mx and L. F. Ramos-deValle, devalle@ciqa.mx

Received 28 May 2012; Revised 12 September 2012; Accepted 17 September 2012

Academic Editor: Theodorian Borca-Tasciuc

Copyright () 2012 F. Avalos-Belmontes et al. This is an open access article distributed under the Creative Commons Attribution License, which permits unrestricted use, distribution, and reproduction in any medium, provided the original work is properly cited.

\begin{abstract}
The effect of carbon nanofibers (CNFs) and carbon nanotubes (CNTs) on the thermal and chemical stability of polypropylene (PP) when subjected to oxidation in a strong acid medium was studied. The effect of CNFs and CNTs on the crystalline morphology and the melting and crystallization temperatures was also studied. The thermal stability increased markedly; the decomposition temperature, for example, increased from $293^{\circ} \mathrm{C}$ for pure PP to 312 and $320^{\circ} \mathrm{C}$ for PP with CNFs and CNTs, respectively. The crystallization temperature increased perceptibly with the addition of CNTs or CNFs, from $107^{\circ} \mathrm{C}$ for pure $\mathrm{PP}$ to 112 and $114^{\circ} \mathrm{C}$ for PP with CNFs and CNTs, respectively. The oxidative degradation with nitric acid produced a reduction in molecular weight; however, this negative effect was less pronounced in the PP compositions with carbon nanoparticles. After 8 hours in nitric acid, this reduction was from 141,000 to 68,000 (for pure PP), to 75,000 (for PP-CNFs), and 79,500 (for PP-CNTs). X-ray diffraction showed that the alpha type crystallinity remains, irrespective of the nucleating agent. Finally, the intensity ratio between the $(040)$ (at $\left.16.7^{\circ}\right)$ and the $(110)\left(\right.$ at $\left.13.9^{\circ}\right)$ reflections increased, which was taken as an indication of an increasing nucleating efficiency.
\end{abstract}

\section{Introduction}

It is well known that polypropylene (PP) is one of the most amply used commodity plastics, mainly because of the ease of processing and the inherent wide range of properties [1]. Additionally, with the addition of filler particles, and especially nanoparticles, this commodity plastic can be tailored to cover many engineering applications. There are many studies that report on the use of different nanoparticles in polypropylene in order to impart or modify a given property. Clay [2-4], calcium carbonate [5], titanium dioxide [6], and carbon nanoparticles [7] have been used to enhance the mechanical and thermal properties, the chemical and flame resistance, and the barrier properties of PP. These nanoparticles may act as nucleating agents when incorporated into $\mathrm{PP}$, promoting thus the heterogeneous crystalline growth.
This would be of utmost interest for applications such as lead-acid car battery cases and geomembranes, where the plastic, mainly $\mathrm{PP}$ or polyethylene (PE), is to be in contact with highly corrosive acid liquids [8]. The chemistry and semicrystallinity characteristics of polyolefins impart to them the good chemical and mechanical properties necessary to assure the extended life of the geomembranes in these applications. Another important application of PP is in leadacid car battery cases in which their outstanding properties make it the ideal option for long time performance.

In both applications the PP is in contact with highly corrosive substances which can alter its composition and structure and significantly reduce its chemical and mechanical properties. In a previous work [7], the authors reported on the excellent nucleating ability of carbon nanoparticles (carbon nanotubes "CNTs" and carbon nanofibers "CNFs"), as compared to commercial nucleating agents for PP. The 
crystallization temperature of $\mathrm{PP}$, from the melt, increased approximately $20^{\circ} \mathrm{C}$ above that of pure $\mathrm{PP}$, using a concentration as low as $0.05 \mathrm{wt} \%$ of carbon nanoparticles. This excellent nucleating capacity of CNTs and CNFs has also been reported by other authors [9-13], though some authors have found that above $4 \mathrm{wt} \%$ this ability reaches a plateau and remains constant [14]. In addition to this nucleating effect, it has been found, via TGA, that these carbon nanoparticles increase the temperature for the onset of the PP decomposition $[12,13,15-17]$ by as much as $20^{\circ} \mathrm{C}$ [18].

In spite of the many reports on the enhancement of the PP properties, conveyed by the nucleating agents in general and by the carbon nanoparticles in particular, very little is known on the effect of these carbon nanoparticles on the chemical stability of PP compositions. The purpose of the present work is to study the relationship between the increase in the thermal and chemical resistance and the enhanced nucleation induced by CNFs, CNTs, and a commercial lithium benzoate (LiBe).

\section{Experimental}

2.1. Materials. The polymer used in this study was a commercial grade isotactic Polypropylene "PP" from Total Petrochemicals, USA, with an average molecular weight Mw of 150,000 and a melt flow rate of $15 \mathrm{~g} / 10 \mathrm{~min}$. The carbon nanoparticles used in this study, were: carbon nanofibers, Pyrograf III (CNFs), also known as StackedCup Carbon Nanotubes (SCCNT), with a nominal surface area of $20 \mathrm{~m}^{2} / \mathrm{g}$, from Applied Sciences, Inc., USA and multi-walled carbon nanotubes (CNTs), with a nominal surface area of $200 \mathrm{~m}^{2} / \mathrm{g}$, from Nano-Lab, Inc., USA. The commercial lithium benzoate (LiBe) nucleating agent was from Micronisers Pty Ltd., Australia. The fuming nitric acid used was from Merck, USA.

2.2. Sample Preparation. A series of PP compositions with $0.05 \mathrm{wt} \%$ of each of CNFs, CNTs, and LiBe were prepared via melt mixing for $10 \mathrm{~min}$, at $170^{\circ} \mathrm{C}$ and $50 \mathrm{rpm}$, in a Brabender torque rheometer mixing chamber. Very low nucleating agent concentrations $(0.05 \mathrm{wt} \%)$ were in order to prevent the excess of nucleating particles from interfering with the spherulitic growth, as has been observed by other authors when using high CNTs or CNFs concentrations $[9,14,19]$. Plain PP was also passed through the mixer to produce blank samples. All compositions were then compression molded to produce $20 \times 20 \times 0.3 \mathrm{~cm}^{3}$ laminates, from which, $10 \mathrm{~cm}$ long and $1 \mathrm{~cm}$ wide strips were cut. These strips were immersed in fuming nitric acid at $80^{\circ} \mathrm{C}$ for periods of 2,8 , and 21 hours, after which, the samples were taken from the nitric acid bath, washed with distilled water until neutral $\mathrm{pH}$, and finally, thoroughly washed with acetone, prior to analysis.

2.3. Evaluation. Following the nitric acid treatment and the appropriate washing, all samples were first analyzed for chemical changes with respect to the time in strong acid medium, using a Perkin-Elmer "Spectrum" FTIR spectrometer with an attenuated total reflectance (ATR) attachment. The spectra were obtained at 20 scans and a resolution of $4 \mathrm{~cm}^{-1}$. Two test specimens were used for each case. The samples were then subjected to thermal analysis using a Perkin-Elmer DSC-7 in order to establish the changes of the thermodynamic variables during treatment in a strong acid medium. Each sample of approximately $8 \mathrm{mg}$ was first heated from room temperature to $180^{\circ} \mathrm{C}$ at $10^{\circ} \mathrm{C} / \mathrm{min}$, held there for $2 \mathrm{~min}$, and then cooled down to room temperature at $10^{\circ} \mathrm{C} / \mathrm{min}$, all under nitrogen atmosphere. In order to study any change in the crystalline morphology, either due to the acid treatment or to the inclusion of nanoparticles, XRD was performed in a Siemens D5000 $(25 \mathrm{~mA}, 35 \mathrm{kV})$ using $\mathrm{CuKa} \mathrm{X}$-ray radiation, at 0.6 degrees/min from 1 to 35 degrees. Finally, the samples were subjected to thermal analysis using a Shimadzu TGA-50 analyzer for studies of the thermal degradation, from room temperature to $400^{\circ} \mathrm{C}$, at a heating rate of $10^{\circ} \mathrm{C} / \mathrm{min}$, under air atmosphere.

A gel permeation chromatograph GPC V-2000, with a refraction index detector and "styragel" columns, was used to determine the evolution of molecular weight and molecular weight distribution with respect to the time of treatment in the strong acid medium. Samples for GPC analysis were first dissolved in trichlorobenzene and then run at $140^{\circ} \mathrm{C}$, at a flow rate of $1 \mathrm{~mL} / \mathrm{min}$.

\section{Results and Discussion}

Figure 1 shows the TGA results of the pure PP and the PP compositions with $0.05 \mathrm{wt} \% \mathrm{LiBe}, \mathrm{CNTs}$, or CNFs, with zero hours of immersion in nitric acid. It can be observed that up to $\sim 220^{\circ} \mathrm{C}$, all 4 samples remain unaltered; but thereafter, the decomposition first occurs in the pure PP, followed by the nucleated compositions; the CNFs and CNTs compounds decompose at the highest temperatures, as shown in Table 1.

These results corroborate the enhanced thermal and chemical stability of the nucleated compositions. This enhanced stability is related to the crystalline structures produced in PP, in the presence of nucleating agents. Miltner et al. and Assouline et al. [19, 20] reported the nucleating ability of carbon nanoparticles and observed that these particles generate polymorphism in PP as well as transcrystallinity in the vicinity of CNTs. It is assumed that this greater molecular order hinders the oxidation of PP.

The increase in the decomposition temperature of the carbon-nucleated compositions may also be due to the barrier effect of the carbon nanoparticles, which when well dispersed, form a barrier that obstructs the oxygen diffusion [21], retarding thus the thermo-oxidative degradation of the polymer. The more marked effect of the CNTs, versus the CNFs, is probably related to the larger nominal surface area.

With respect to the decomposition of PP in nitric acid, Figure 2 shows the evolution of $\mathrm{NO}_{2}$ and $\mathrm{CO}$ with time, on samples immersed in nitric acid for 8 and 22 hours, as taken from the IR absorbance peaks at 1555 and $1710 \mathrm{~cm}^{-1}$, respectively. It is observed that as the immersion time in nitric acid increases, the presence of $\mathrm{NO}_{2}$ increases, becoming apparent after 8 hours in nitric acid and increasing 


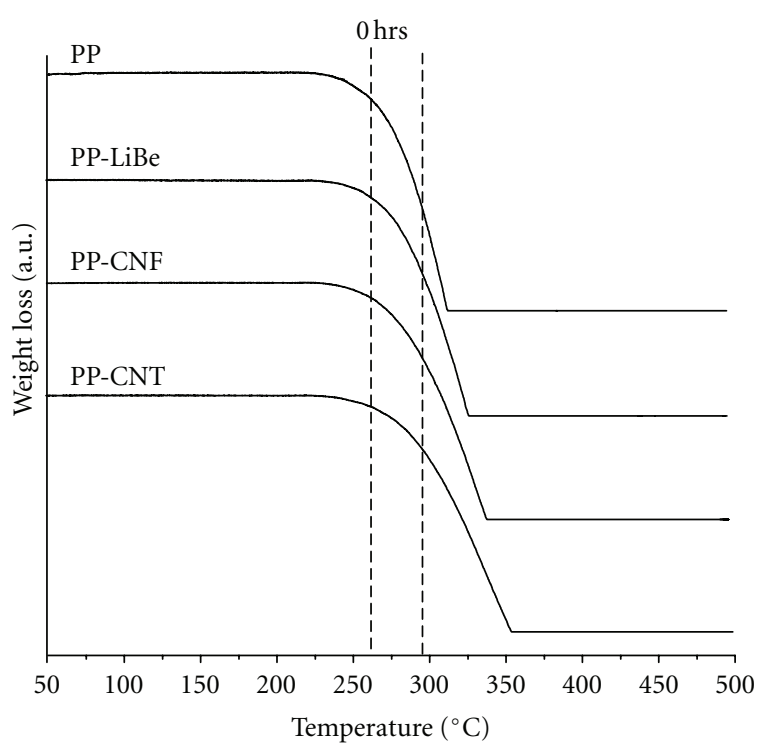

Figure 1: TGA curves showing the weight loss with temperature, due to decomposition of pure PP and PP compositions with $0.05 \mathrm{wt} \%$ of nucleating agent. Samples without immersion in nitric acid. The dotted lines represent the temperatures at which pure PP weight loss reached 10 and $50 \mathrm{wt} \%$.

its presence with time. The presence of $\mathrm{CO}$ on the other hand becomes apparent only after 22 hours in nitric acid.

The decomposition first involves the nitration of the PP chain, prior to the chain scission that starts to occur as a result of the formation of carbonyl groups. This is why the carbonyl bands in the FTIR analysis appear only after 22 hours in nitric acid [22]. This indicates that the PP oxidation in acid medium, quite different to that reported for PE [21], could be assumed to be due to the presence of the labile hydrogen atoms in the PP methyl groups. The greater concentration of $\mathrm{NO}_{2}$ in the compositions with CNFs or CNTs is simply because these nanoparticles tend to oxidize in strong acid medium [23-25].

GPC results, showing the decrease in molecular weight of the pure PP samples with the time of immersion in nitric acid are presented in Figure 3(a). It is observed that after 2 hours in nitric acid, the decrease in molecular weight, (molecular weight taken at the peak of the GPC curves and designated as "Mp"), was negligible and remained at $\sim 141,000$ (i.e., $\left.10^{5.15}\right)$. But after 8 hours in nitric acid, this decrease in $\mathrm{Mp}$ was considerable from $\sim 141,000$ to $\sim 68,000$ (i.e., $10^{4.83}$ ).

Figure 3(b), on the other hand, shows that after 8 hours in nitric acid, the molecular weight, as taken at the peak of the GPC curve, decreased from 141,000, for the original $\mathrm{PP}$, to 68,000 ( $\left.\mathrm{PP}_{\text {Pure }}\right), 71,000\left(\mathrm{PP}_{\mathrm{LiBe}}\right), 75,000\left(\mathrm{PP}_{\mathrm{CNFs}}\right)$, and $79,500\left(\mathrm{PP}_{\mathrm{CNTs}}\right)$. The differences in molecular weight after the 8 hours in nitric acid are really small. These small differences are assumed to arise because the nucleated crystalline structures provide a slightly better chemical stability for the PP nanocompounds. In addition, the carbon nanoparticles tend to act as free radical scavengers, as reported elsewhere $[12,13,15,23]$, further retarding a little bit the PP decrease

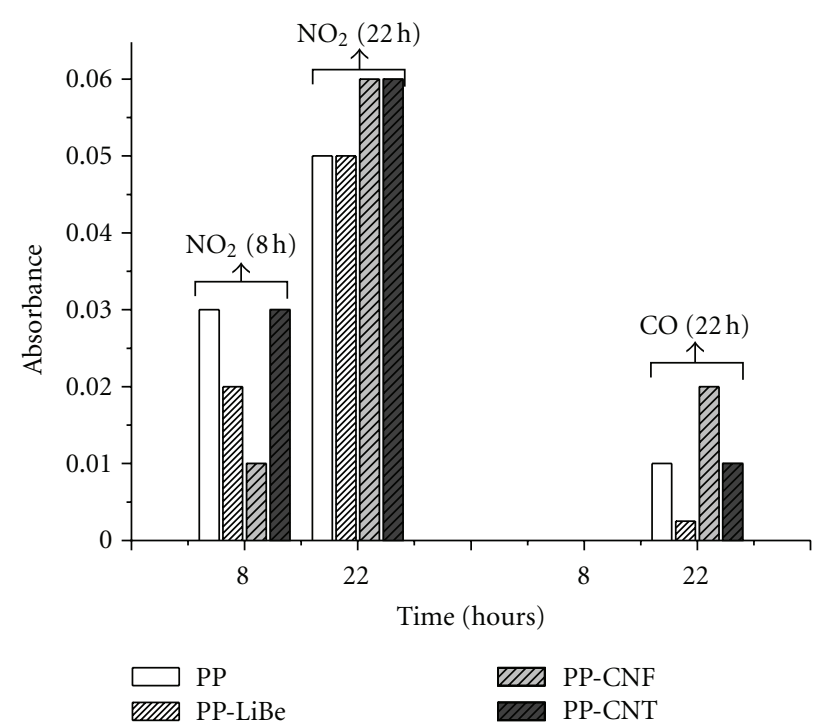

Figure 2: Variation of $\mathrm{CO}$ and $\mathrm{NO}_{2}$ groups with the time of immersion in nitric acid, as taken from the peaks at $1555\left(\mathrm{NO}_{2}\right)$ and $1710(\mathrm{CO}) \mathrm{cm}^{-1}$ in the IR absorbance diagrams.

TABle 1: Temperatures at which there was a 10 and $50 \mathrm{wt} \%$ loss, due to chemical decomposition, in PP samples collected after immersion in nitric acid for 0 and 8 hours.

\begin{tabular}{lccc}
\hline & & $\begin{array}{c}\mathrm{T}\left({ }^{\circ} \mathrm{C}\right) \text { at } \\
10 \mathrm{wt} \% \text { loss }\end{array}$ & $\begin{array}{c}\mathrm{T}\left({ }^{\circ} \mathrm{C}\right) \text { at } \\
50 \mathrm{wt} \% \text { loss }\end{array}$ \\
\hline \multirow{4}{*}{ 0 hours in nitric acid } & PP-Pure & 263 & 293 \\
& PP-LiBe & 268 & 304 \\
& PP-CNFs & 272 & 312 \\
& PP-CNTs & 277 & 320 \\
\hline \multirow{4}{*}{ After 8 hours in nitric } & PP-Pure & 303 & 365 \\
acid & PP-LiBe & 316 & 379 \\
& PP-CNFs & 319 & 381 \\
& PP-CNTs & 321 & 387 \\
\hline
\end{tabular}

in molecular weight. The degradation mechanism is assumed to involve the separation of the labile hydrogen atoms and their substitution by the $\mathrm{NO}_{2}$ groups in the amorphous phase of the main PP chain, followed by the chain scission. This results in the terminal carbonyl groups, as observed via the FTIR analysis.

Figure 4 shows the TGA curves of pure PP and PP compositions with $0.05 \mathrm{wt} \% \mathrm{LiBe}, \mathrm{CNTs}$, or CNFs obtained in samples collected after 8 hours of immersion in nitric acid. First, it can be observed that all 4 samples remain unaltered up to $\sim 240^{\circ} \mathrm{C}$, that is, $20^{\circ} \mathrm{C}$ higher than that for samples with 0 hours in nitric acid (Figure 1). But thereafter, again, the decomposition first occurs in the pure PP, followed by the LiBe, CNFs, and CNTs compounds, as shown in Table 1.

Comparing the results among samples immersed in nitric acid for 0 and 8 hours (Figures 1 and 4, resp.), it can be observed that samples immersed in nitric acid (Figure 4), decompose at much higher temperatures, showing a 10 and 50 weight percent loss at temperatures $\sim 42$ and $\sim 70^{\circ} \mathrm{C}$ 


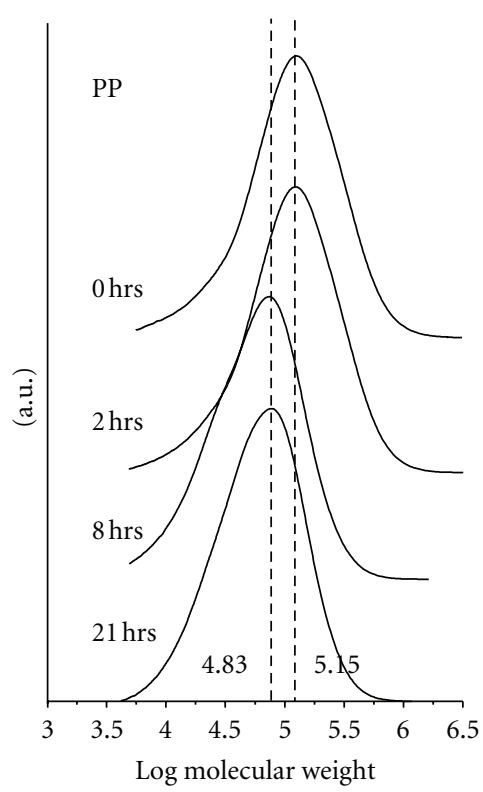

(a)

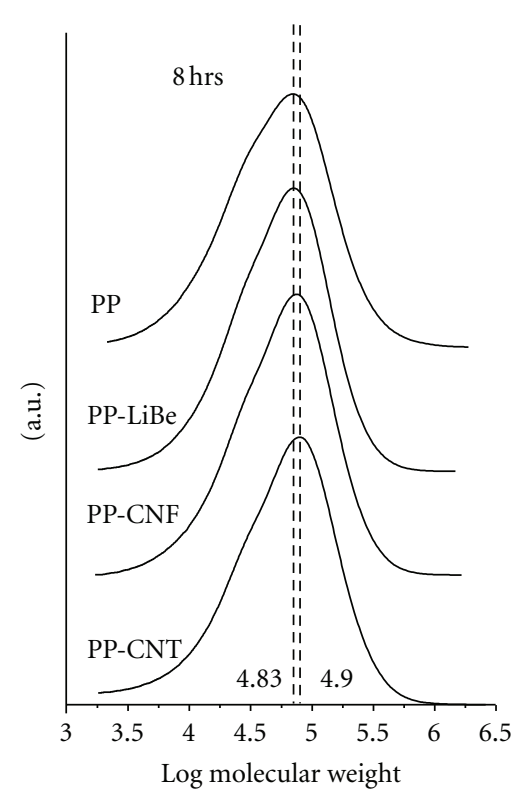

(b)

FIGURE 3: (a) GPC curves showing the variation of molecular weight with the time of immersion in nitric acid for pure PP and (b) showing the differences in molecular weight between the nonnucleated and nucleated PP's, of samples collected after 8 hours in nitric acid.

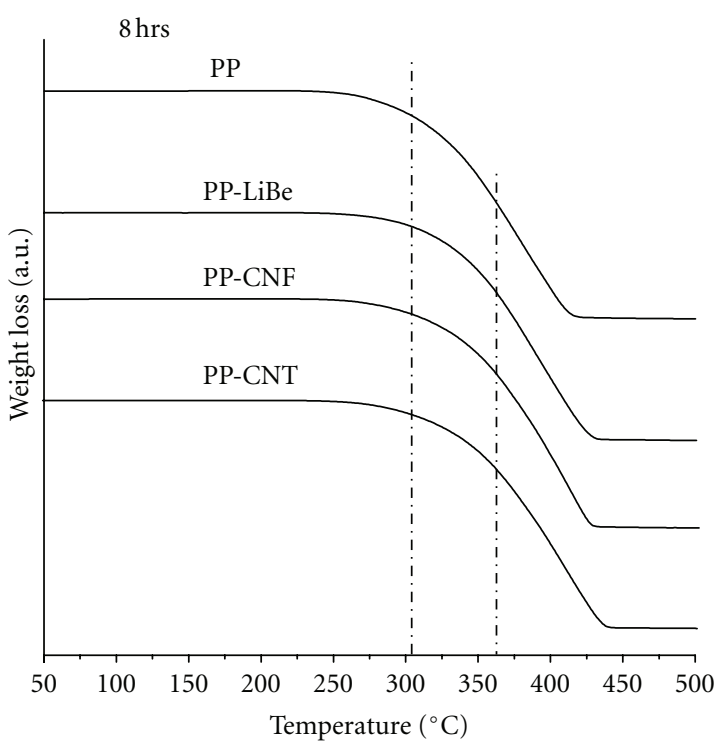

FIgURE 4: TGA curves showing the weight loss with temperature due to decomposition of pure PP and PP compositions with $0.05 \mathrm{wt} \%$ of nucleating agent. Samples collected after 8 hours of immersion in nitric acid. The dotted lines represent the temperatures at which pure PP weight loss reached 10 and $50 \mathrm{wt} \%$.

higher, respectively, than those corresponding to samples without immersion in nitric acid (Figure 1).

Considering that the immersion in nitric acid tends to wash out the amorphous fraction of the PP samples, then, the immersed samples in Figure 4 surely have a greater crystalline fraction than the not immersed samples in Figure 1, producing thus the marked differences in thermal stability, which could be attributed entirely to the greater crystalline fraction.

The XRD diffractograms in Figure 5 show the position of the crystalline planes of pure PP and of the nucleated PP, with 0 and 8 hours of immersion in nitric acid. It can be observed that neither the nucleating agents nor the immersion in nitric acid have an effect on the position of the diffraction signals.

This indicates that the crystalline type remains the same alpha crystals. Both, pure PP and PP compositions with nucleating agent, either with 0 or with 8 hours of immersion in nitric acid, present the same peaks at 2(theta), that is, $13.9,16.7,18.3,21.6$, and 21.9 degrees, which correspond to the planes (110), (040), (130), (111), and (041), respectively, of this alpha morphology. These results coincide with those found for PP/SWNT and PP/MWNT, as reported by several authors [26-28].

In addition, a clear tendency for the increase of the intensity ratio between the (040) (16.7 degrees) and the (110) (13.9 degrees) reflections can be observed. This increase can be taken as an indication of an increasing nucleating efficiency, as reported elsewhere [29].

Figure 6 shows the fusion thermograms of the different PP samples, where it can be observed that the peak fusion temperatures are very similar among them, $150.6^{\circ} \mathrm{C}$ for pure $\mathrm{PP}$ and $151.5^{\circ} \mathrm{C}$ for the nucleated PP compositions.

However, the fusion interval appears to be narrower for the PP compositions with nucleating agent, LiBe, CNTs, or CNFs, surely due to the greater crystal size homogeneity.

In addition, it is observed in the inserted Table that the enthalpy of fusion increases very slightly in the nucleated samples. Considering thus the very small enthalpy changes among the PP and the PP with the different nucleating agents, it can be concluded that the percent crystallinity remains approximately the same. 


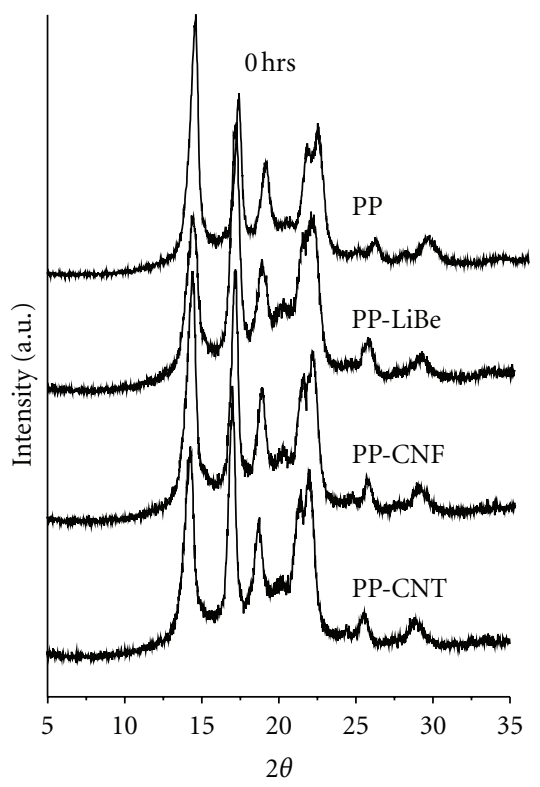

(a)

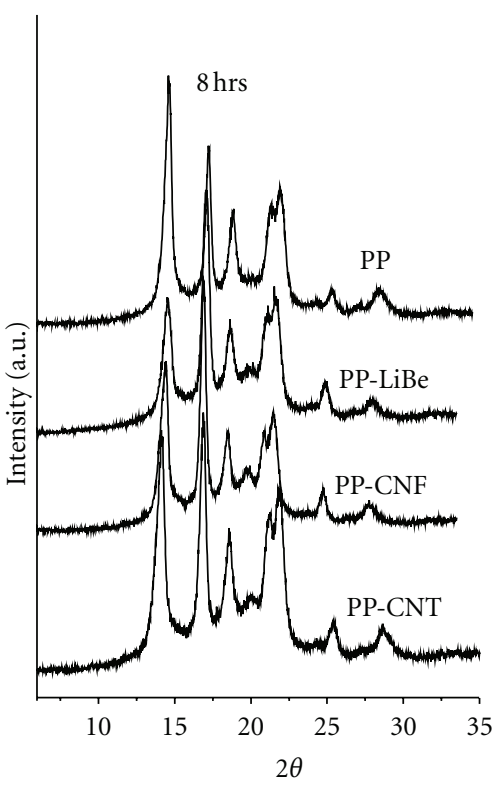

(b)

FIGURE 5: XRD diffractograms of pure PP and PP compositions with $0.05 \mathrm{wt} \%$ of nucleating agent; (a) without immersion in nitric acid and (b) after 8 hours in nitric acid.

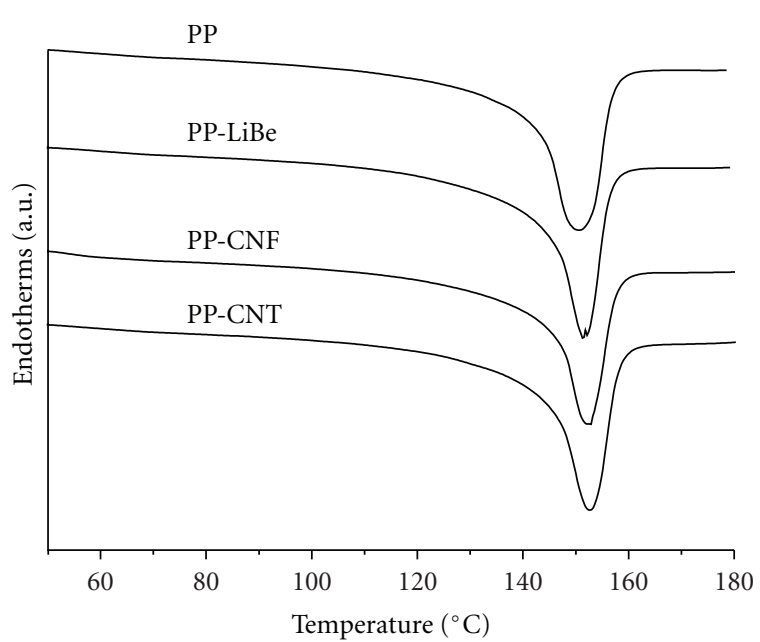

Figure 6: DSC fusion thermograms of pure PP and PP compositions with $0.05 \mathrm{wt} \%$ of nucleating agent, without immersion in nitric acid.

Figures $7(\mathrm{a})$ and $7(\mathrm{~b})$ present the variation of the crystallization and fusion temperatures, respectively, as obtained in a DSC experiment for pure PP and the PP compositions with LiBe, CNTs, and CNFs, in samples collected after different times of immersion in nitric acid. Figure 7(a) shows that $T_{c}$ increased from $107^{\circ} \mathrm{C}$ for the PP to 110,112 , and $114^{\circ} \mathrm{C}$, for the PP compositions with LiBe, CNFs, and CNTs, respectively. It is thus apparent, that the most efficient nucleating agents are the CNTs, followed closely by the CNFs. The slight difference among them is attributed to the greater nominal surface area of the CNTs. Figure $7(b)$, on the other hand, shows that $T_{m}$ is around $151 \pm 1^{\circ} \mathrm{C}$ in samples collected after 0 and 2 hours in nitric acid, but then decreases after 8 and 21 hours in nitric acid. It is also observed that, for all times of immersion in nitric acid, the nucleated PP compositions, especially those with CNFs or CNTs, present a higher $T_{m}$ than that of pure PP. This confirms the greater nucleating efficiency of the CNFs and CNTs, as shown in Figure 8.

The nucleating efficiency (NE) $[30,31]$ was calculated using (1) as follows:

$$
\mathrm{NE}=100 \frac{T_{c}-T_{c 1}}{T_{c 2 \max }-T_{c 1}},
$$

where $T_{c}$ is the measured crystallization temperature for the particular nucleating agent, and $T_{c 1}$ and $T_{c 2 \max }$ are the crystallization temperatures of the nonnucleated and selfnucleated polymers, respectively. A value of $T_{c 2 \max }$ equal to $140^{\circ} \mathrm{C}$ was obtained from the self-nucleation experiments. The crystallization time $t_{c}$, was calculated using (2) as follows:

$$
t_{c}=\frac{T_{\text {onset }}-T_{c}}{R_{c}},
$$

where $R_{c}$ is the rate of cooling in the DSC crystallization experiment $\left({ }^{\circ} \mathrm{C} / \mathrm{min}\right)$, and $T_{\text {onset }}$ and $T_{c}$ are the temperatures at the onset of crystallization and at the maximum rate of crystallization (i.e., at the peak of the DSC crystallization curve) [32]. These data is presented in Figure 8, where it can be observed that the most efficient nucleating agents are the CNTs, followed by the CNFs; but it can also be observed that pure PP presents an apparent negative nucleating efficiency, which could be attributed to the presence of grafted chemical groups on the main PP chain after the nitric acid treatment. The crystallization time is approximately the same for the 3 


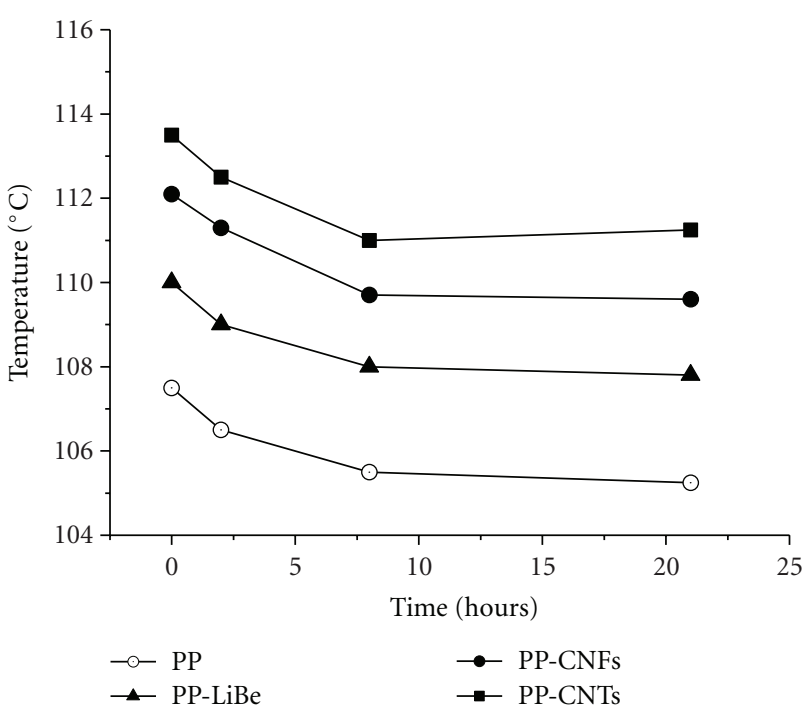

(a) $T_{c}$

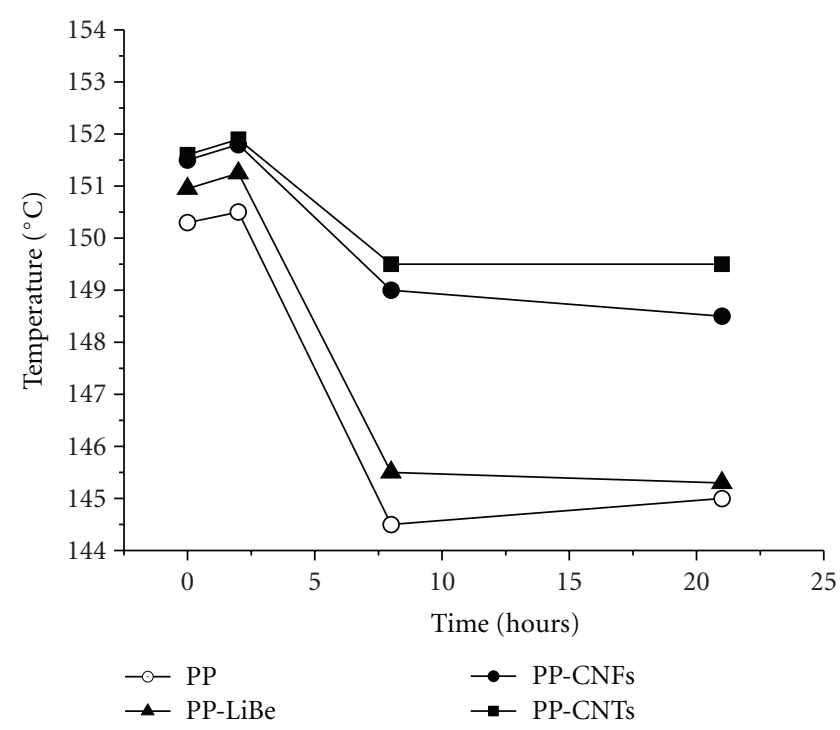

(b) $T_{m}$

FIGURE 7: Variation of the crystallization (a) and melting (b) peak temperatures, as obtained from DSC, for pure PP and PP compositions with $0.05 \mathrm{wt} \%$ of nucleating agent, after immersion in nitric acid for different times.

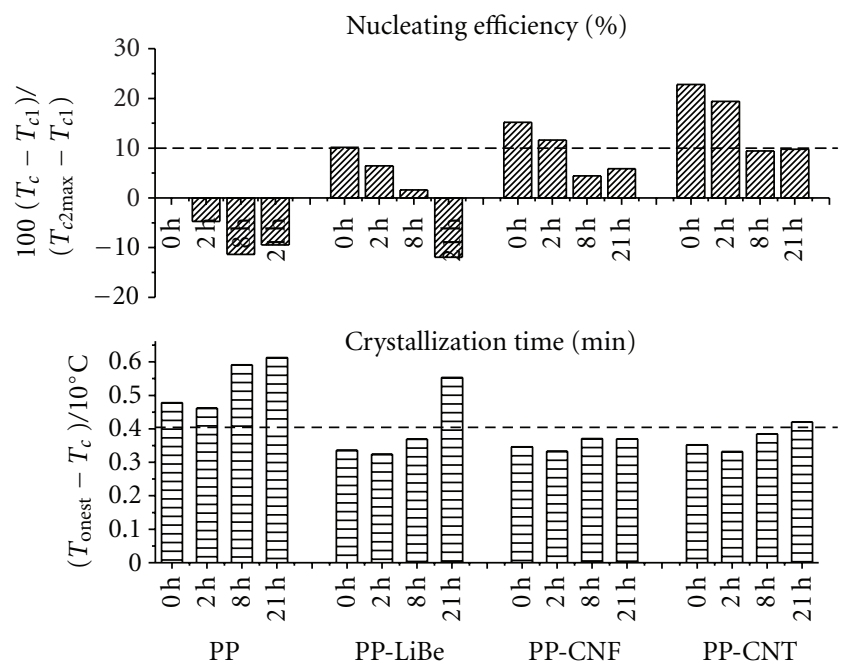

Figure 8: Nucleating efficiency (NE) and crystallization time $t_{c}$, calculated using (1) and (2), respectively; for pure PP and PP compositions with 0.05 wt $\%$ of LiBe, CNTs and CNFs, after immersion in nitric acid for different times.

nucleating agents, that is, LiBe, CNFs, and CNTs; but this is markedly less than that for pure PP.

It is assumed that the greater nucleating efficiency of CNFs and CNTs, as observed in Figure 8, is the main reason for the slightly better thermal and chemical stability of the corresponding PP compounds, as obtained from the TGA and GPC analyses, and of the increased $T_{m}$ and $T_{c}$, as obtained from the DSC analyses.

Finally, considering that the amount and morphology of the crystalline structure in a semicrystalline polymer greatly affects in a positive way its chemical and mechanical properties, the inclusion of carbon nanoparticles as nucleating agents in PP would ensure better chemical resistance and mechanical properties when subjected to aggressive environments along its useful life.

\section{Conclusions}

Carbon nanoparticles, such as CNTs and CNFs increased markedly the thermal and chemical properties of PP, acting as very efficient nucleating agents, even better than the commercial LiBe, The decomposition temperature increased from $293^{\circ} \mathrm{C}$ for the pure PP to 312 and $320^{\circ} \mathrm{C}$, for the PP compositions with CNFs and CNTs, respectively. The oxidative degradation with nitric acid produced a reduction in molecular weight, as determined by DSC; however, this was much less pronounced in the PP compositions with carbon nanoparticles. This reduction was from 141,000 to 68,000, 75,000, and 79,500, for pure PP, PP-CNFs, and PPCNTs, respectively.

XRD results showed that the crystalline type was not affected by the nucleating agent; it remained of the alpha type. In addition, it was found that the carbon nanoparticles are highly efficient nucleating agents for the crystallization of PP. The crystallization temperature (DSC) and the decomposition temperature (TGA) of PP, both increased with the addition of CNTs or CNFs.

In general the mechanical performance of $\mathrm{PP}$ composites can be enhanced by the addition of CNTs or CNFs as nucleating agents. This increased resistance of $\mathrm{PP} /$ carbon nanoparticles compositions to thermal and chemical attack increases the viability of using PP in such demanding applications as lead-acid car battery cases and films to be used as geomembranes.

Considering that the amount and morphology of the crystalline structure in a semicrystalline polymer greatly 
affects in a positive way its chemical and mechanical properties, it can be concluded that the inclusion of carbon nanoparticles as nucleating agents in PP would ensure better thermal and chemical resistance when subjected to aggressive environments.

\section{Acknowledgments}

The authors wish to thank CONACYT for its financial support to carry out this study, through Project 84424. In addition, one of the authors F. Avalos-Belmontes wishes to thank CONACYT for its support to spend a sabbatical at CIQA. Finally, the authors wish to thank M. Sánchez-Adame, A. Espinoza-Muñoz, M. C. Gonzalez-Cantu, T. RodriguezHernandez, B. Huerta-Martinez, J. Rodriguez-Velazquez, J. F. Zendejo, E. Hurtado-Suarez, and P. Siller-Flores for their valuable technical and informatics support.

\section{References}

[1] H. G. Karian, Ed., Handbook of Polypropylene and Polypropylene Composites, CRC Press, New York, NY, USA, 2nd edition, 2003.

[2] G. Galgali, S. Agarwal, and A. Lele, "Effect of clay orientation on the tensile modulus of polypropylene-nanoclay composites," Polymer, vol. 45, no. 17, pp. 6059-6069, 2004.

[3] G. Mani, Q. Fan, S. C. Ugbolue, and Y. Yang, "Morphological studies of polypropylene-nanoclay composites," Journal of Applied Polymer Science, vol. 97, no. 1, pp. 218-226, 2005.

[4] B. K. Kandola, G. Smart, A. R. Horrocks et al., "Effect of different compatibilisers on nanoclay dispersion, thermal stability, and burning behavior of polypropylene-nanoclay blends," Journal of Applied Polymer Science, vol. 108, no. 2, pp. 816-824, 2008.

[5] T. Zaharescu, S. Jipa, W. Kappel, and P. Supaphol, "The control of thermal and radiation stability of polypropylene containing calcium carbonate nanoparticles," Macromolecular Symposia, vol. 242, pp. 319-324, 2006.

[6] Q. Wei, L. Yu, R. R. Mather, and X. Wang, "Preparation and characterization of titanium dioxide nanocomposite fibers," Journal of Materials Science, vol. 42, no. 19, pp. 8001-8005, 2007.

[7] S. Reyes-De Vaaben, A. Aguilar, F. Avalos, and L. F. Ramos-De Valle, "Carbon nanoparticles as effective nucleating agents for polypropylene," Journal of Thermal Analysis and Calorimetry, vol. 93, no. 3, pp. 947-952, 2008.

[8] P. A. Dilara and D. Briassoulis, "Degradation and stabilization of low-density polyethylene films used as greenhouse covering materials," Journal of Agricultural Engineering Research, vol. 76, no. 4, pp. 309-321, 2000.

[9] A. Funck and W. Kaminsky, "Polypropylene carbon nanotube composites by in situ polymerization," Composites Science and Technology, vol. 67, no. 5, pp. 906-915, 2007.

[10] H. Xia, Q. Wang, K. Li, and G. H. Hu, "Preparation of polypropylene/carbon nanotube composite powder with a solid-state mechanochemical pulverization process," Journal of Applied Polymer Science, vol. 93, no. 1, pp. 378-386, 2004.

[11] K. Lu, N. Grossiord, C. E. Koning, H. E. Miltner, B. Van Mele, and J. Loos, "Carbon nanotube/isotactic polypropylene composites prepared by latex technology: morphology analysis of CNT-induced nucleation," Macromolecules, vol. 41, no. 21, pp. 8081-8085, 2008.
[12] S. L. Kodjie, L. Li, B. Li, W. Cai, C. Y. Li, and M. Keating, "Morphology and crystallization behavior of HDPE/CNT nanocomposite," Journal of Macromolecular Science, vol. 45, no. 2, pp. 231-245, 2006.

[13] G. Sui, W. H. Zhong, M. A. Fuqua, and C. A. Ulven, "Crystalline structure and properties of carbon nanofiber composites prepared by melt extrusion," Macromolecular Chemistry and Physics, vol. 208, no. 17, pp. 1928-1936, 2007.

[14] Y. Peneva, M. Valcheva, L. Minkova, M. Mičušík, and M. Omastová, "Nonisothermal crystallization kinetics and microhardness of PP/CNT composites," Journal of Macromolecular Science B, vol. 47, no. 6, pp. 1197-1210, 2008.

[15] P. C. P. Watts, P. K. Fearon, W. K. Hsu, N. C. Billingham, H. W. Kroto, and D. R. M. Walton, "Carbon nanotubes as polymer antioxidants," Journal of Materials Chemistry, vol. 13, no. 3, pp. 491-495, 2003.

[16] B. B. Marosfoi, A. Szabó, G. Marosi, D. Tabuani, G. Camino, and S. Pagliari, "Thermal and spectroscopic characterization of polypropylene-carbon nanotube composites," Journal of Thermal Analysis and Calorimetry, vol. 86, no. 3, pp. 669-673, 2006.

[17] A. Chatterjee and B. L. Deopura, "Thermal stability of polypropylene/carbon nanofiber composite," Journal of Applied Polymer Science, vol. 100, no. 5, pp. 3574-3578, 2006.

[18] M. M. Hasan, Y. Zhou, and S. Jeelani, "Thermal and tensile properties of aligned carbon nanofiber reinforced polypropylene," Materials Letters, vol. 61, no. 4-5, pp. 1134-1136, 2007.

[19] H. E. Miltner, N. Grossiord, K. Lu, J. Loos, C. E. Koning, and B. Van Mele, "Isotactic polypropylene/carbon nanotube composites prepared by latex technology. Thermal analysis of camon nanotube induced nucleation," Macromolecules, vol. 41, no. 15 , pp. 5753-5762, 2008.

[20] E. Assouline, A. Lustiger, A. H. Barber et al., "Nucleation ability of multiwall carbon nanotubes in polypropylene composites," Journal of Polymer Science B, vol. 41, no. 5, pp. 520527, 2003.

[21] N. Khelidj, X. Colin, L. Audouin, J. Verdu, C. Monchy-Leroy, and V. Prunier, "Oxidation of polyethylene under irradiation at low temperature and low dose rate. Part I. the case of "pure" radiochemical initiation," Polymer Degradation and Stability, vol. 91, no. 7, pp. 1593-1597, 2006.

[22] H. Tanaka, "Fuming nitric acid treatment of isotactic polypropylene film, I: effects of selective oxidation on proton spin-lattice and spin-spin relaxation times," Colloid \& Polymer Science, vol. 260, no. 12, pp. 1101-1106, 1982.

[23] I. D. Rosca, F. Watari, M. Uo, and T. Akasaka, "Oxidation of multiwalled carbon nanotubes by nitric acid," Carbon, vol. 43, no. 15, pp. 3124-3131, 2005.

[24] S. Armenise, M. Nebra, E. García-Bordejé, and A. Monzón, "Functionalization of carbon nanofibers coated on cordierite monoliths by oxidative treatment," Studies in Surface Science and Catalysis, vol. 175, pp. 483-486, 2010.

[25] P. V. Lakshminarayanan, H. Toghiani, and C. U. Pittman, "Nitric acid oxidation of vapor grown carbon nanofibers," Carbon, vol. 42, no. 12-13, pp. 2433-2442, 2004.

[26] E. Logakis, E. Pollatos, C. Pandis et al., "Structure-property relationships in isotactic polypropylene/multi-walled carbon nanotubes nanocomposites," Composites Science and Technology, vol. 70, no. 2, pp. 328-335, 2010.

[27] A. R. Bhattacharyya, T. V. Sreekumar, T. Liu et al., "Crystallization and orientation studies in polypropylene/single wall carbon nanotube composite," Polymer, vol. 44, no. 8, pp. 2373-2377, 2003. 
[28] B. K. Satapathy, M. Ganß, R. Weidisch et al., "Ductileto-semiductile transition in PP-MWNT nanocomposites," Macromolecular Rapid Communications, vol. 28, no. 7, pp. 834-841, 2007.

[29] E. Ferrage, F. Martin, A. Boudet et al., "Talc as nucleating agent of polypropylene: morphology induced by lamellar particles addition and interface mineral-matrix modelization," Journal of Materials Science, vol. 37, no. 8, pp. 1561-1573, 2002.

[30] B. Fillon, A. Thierry, B. Lotz, and J. C. Wittmann, "Efficiency scale for polymer nucleating agents," Journal of Thermal Analysis, vol. 42, no. 4, pp. 721-731, 1994.

[31] C. Marco, G. Ellis, M. A. Gómez, and J. M. Arribas, "Analysis of the dynamic crystallisation of isotactic polypropylene/ $\alpha$ nucleating agent systems by DSC," Journal of Thermal Analysis and Calorimetry, vol. 68, no. 1, pp. 61-74, 2002.

[32] M. A. Islam, I. A. Hussein, and M. Atiqullah, "Effects of branching characteristics and copolymer composition distribution on non-isothermal crystallization kinetics of metallocene LLDPEs," European Polymer Journal, vol. 43, no. 2, pp. 599-610, 2007. 

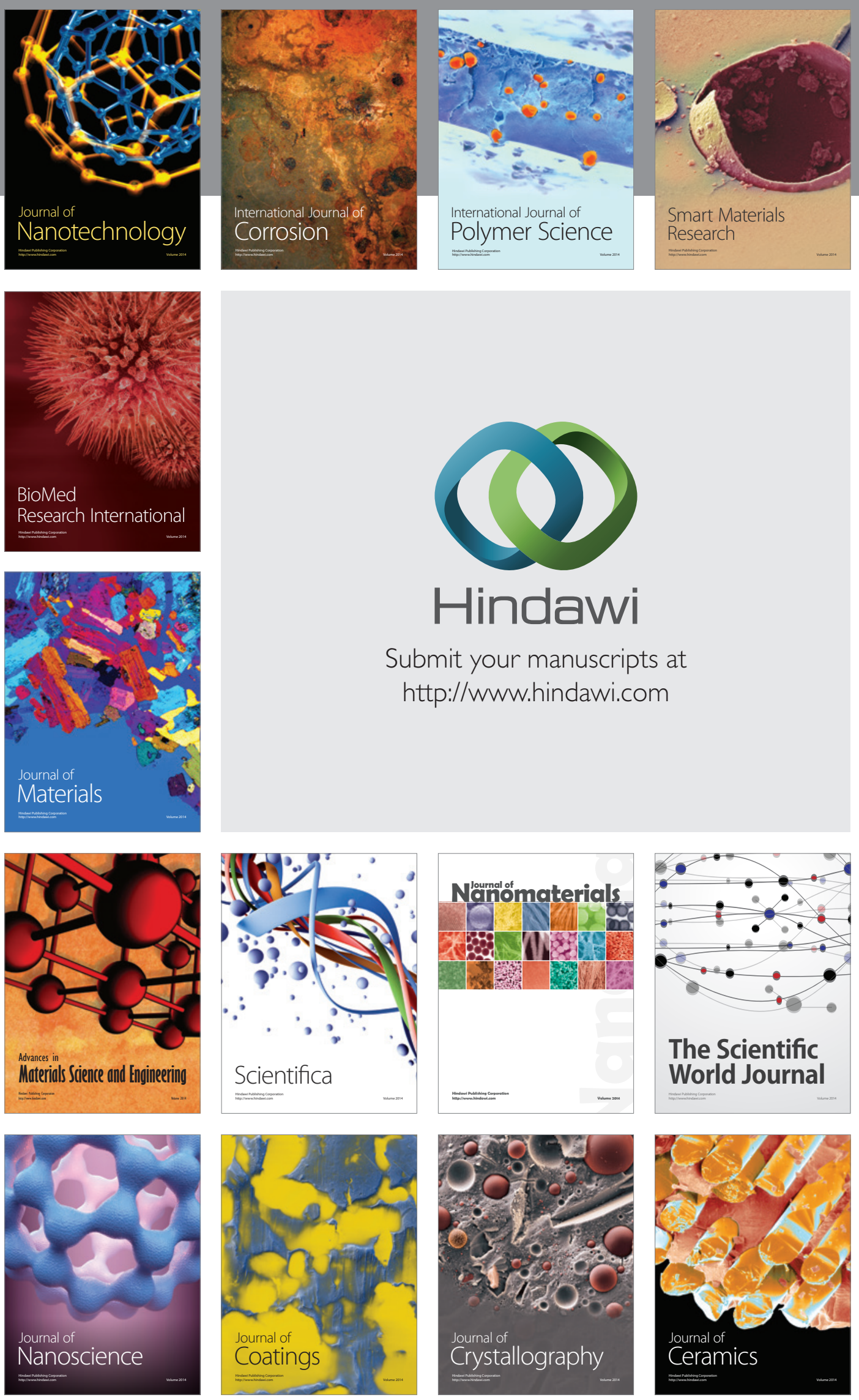

The Scientific World Journal

Submit your manuscripts at

http://www.hindawi.com

\section{World Journal}

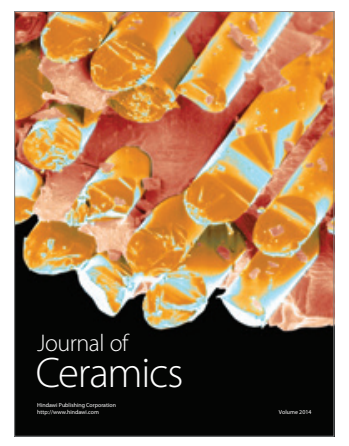

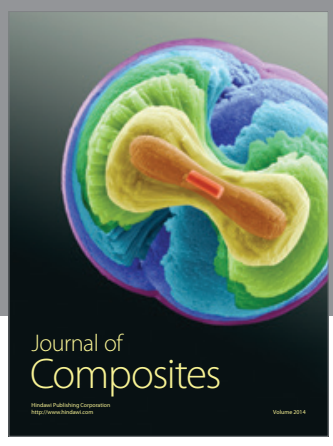
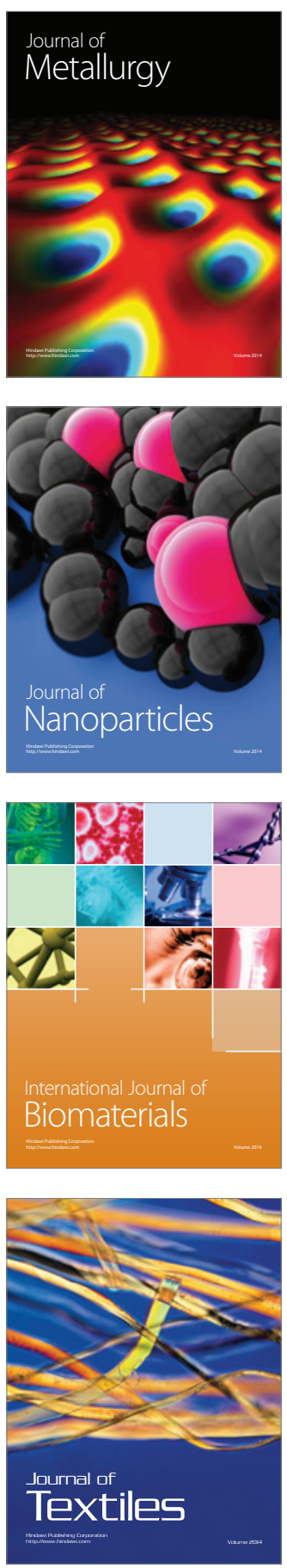